\title{
NONLINEAR CONTRACTIONS IN METRIC SPACES UNDER LOCALLY T-TRANSITIVE BINARY RELATIONS
}

\author{
AFTAB ALAM* AND MOHAMMAD IMDAD** \\ *Department of Mathematics, Aligarh Muslim University, Aligarh-202002, India \\ E-mail: aafu.amu@gmail.com \\ **Department of Mathematics, Aligarh Muslim University, Aligarh-202002, India \\ E-mail: mhimdad@gmail.com
}

\begin{abstract}
In this paper, we present a variant of Boyd-Wong fixed point theorem in a metric space equipped with a locally $T$-transitive binary relation, which under universal relation reduces to Boyd-Wong (Proc. Amer. Math. Soc. 20(1969), 458-464) and Jotic (Indian J. Pure Appl. Math. 26(1995), 947-952) fixed point theorems. Also, our results extend several other well-known fixed point theorems such as: Alam and Imdad (J. Fixed Point Theory Appl. 17(2015), no 4, 693-702) and Karapinar and Roldán-López-de-Hierro (J. Inequal. Appl. 2014:522(2014), 12 pages) besides some others.

Key Words and Phrases: $\mathcal{R}$-continuity; locally $T$-transitive binary relations; $\varphi$-contractions;
\end{abstract} $\mathcal{R}$-connected sets; fixed point.

2010 Mathematics Subject Classification: 47H10, 54H25.

Acknowledgements. Both the authors are grateful to an anonymous learned referee for his fruitful comments and valuable suggestions specially pointing out an error in the earlier proof. The authors are also grateful to Professor Mihai Turinici for providing the file of his book chapter. The first author is thankful to University Grant Commission, New Delhi, Government of India for awarding BSR (Basic Scientific Research) Fellowship.

\section{REFERENCES}

[1] A. Alam, A.R. Khan, M. Imdad, Some coincidence theorems for generalized nonlinear contractions in ordered metric spaces with applications, Fixed Point Theory Appl., 216(2014), 30 pages.

[2] A. Alam, M. Imdad, Relation-theoretic contraction principle, J. Fixed Point Theory Appl., $\mathbf{1 7}(2015)$, no. 4, 693-702.

[3] A. Alam, M. Imdad, Relation-theoretic metrical coincidence theorems with applications, Filomat (in press).

[4] S. Banach, Sur les operations dans les ensembles abstraits et leur application aux equations intgrales, Fund. Math., 3(1922), 133-181.

[5] M. Berzig, E. Karapinar, A. Roldán, Discussion on generalized- $(\alpha \psi, \beta \varphi)$-contractive mappings via generalized altering distance function and related fixed point theorems, Abstr. Appl. Anal., $\mathbf{2 5 9 7 6 8 ( 2 0 1 4 ) , ~} 12$ pages. 
[6] D.W. Boyd, J.S.W. Wong, On nonlinear contractions, Proc. Amer. Math. Soc., 20(1969), 458464.

[7] R. DeMarr, Common fixed points for isotone mappings, Colloq. Math., 13(1964), 45-48.

[8] N. Jotic, Some fixed point theorems in metric spaces, Indian J. Pure Appl. Math., 26(1995), 947-952.

[9] E. Karapinar, A. Roldán, N. Shahzad, W. Sintunavarat, Discussion of coupled and tripled coincidence point theorems for $\varphi$-contractive mappings without the mixed $g$-monotone property, Fixed Point Theory Appl., 92(2014), 16 pages.

[10] E. Karapinar, A.F. Rolán-López-de-Hierro, A note on ' $(G, F)$-Closed set and tripled point of coincidence theorems for generalized compatibility in partially metric spaces', J. Inequal. Appl., 522(2014), 12 pages.

[11] B. Kolman, R.C. Busby, S. Ross, Discrete mathematical structures, Third Edition, PHI Pvt. Ltd., New Delhi, 2000.

[12] M.A. Kutbi, A. Roldán, W. Sintunavarat, J. Martinez-Moreno, C. Roldan, F-closed sets and coupled fixed point theorems without the mixed monotone property, Fixed Point Theory Appl., $\mathbf{3 3 0}(2013)$, 11 pages.

[13] V. Lakshmikantham, L. Cirić, Coupled fixed point theorems for nonlinear contractions in partially ordered metric spaces, Nonlinear Anal., 70(2009), 4341-4349.

[14] S. Lipschitz, Schaum's Outlines of Theory and Problems of Set Theory and Related Topics, McGraw-Hill, New York, 1964.

[15] E.J. McShane, Order-preserving Maps and Integration Processes, Ann. Math. St. Vol. 31, Princeton Univ. Press, Princeton, 1953.

[16] J.J. Nieto, R. Rodríguez-López, Contractive mapping theorems in partially ordered sets and applications to ordinary differential equations, Order, 22(2005), no. 3, 223-239.

[17] A.F. Roldán-López-de-Hierro, E. Karapinar, M. de la Sen, Coincidence point theorems in quasimetric spaces without assuming the mixed monotone property and consequences in G-metric spaces, Fixed Point Theory Appl., 184(2014), 29 pages.

[18] B. Samet, E. Karapinar, H. Aydi, V.C. Rajic, Discussion on some coupled fixed point theorems, Fixed Point Theory Appl., 50(2013), 12 pages.

[19] B. Samet, M. Turinici, Fixed point theorems on a metric space endowed with an arbitrary binary relation and applications, Commun. Math. Anal., 13(2)(2012), 82-97.

[20] B. Samet, C. Vetro, Coupled fixed point, F-invariant set and fixed point of N-order, Ann. Funct. Anal., 1(2010), no. 2, 46-56.

[21] W. Sintunavarat, P. Kumam, Y.J. Cho, Coupled fixed point theorems for nonlinear contractions without mixed monotone property, Fixed Point Theory Appl., 170(2012), 16 pages.

[22] A. Tarski, A lattice-theoretical fixpoint theorem and its applications, Pacific J. Math., 5(1955), 285-309.

[23] M. Turinici, Product fixed points in ordered metric spaces, arXiv:1110.3079v1, 2011.

[24] M. Turinici, Linear contractions in product ordered metric spaces, Ann. Univ. Ferrara, 59(2013), 187-198

[25] M. Turinici, Contractive maps in locally transitive relational metric spaces, The Sci. World J., 169358(2014), 10 pages.

[26] M. Turinici, Contractive operators in relational metric spaces, Handbook of Functional Equations, Springer Optimization and its Applications, Springer, Vol. 95, 2014, 419-458.

[27] J. Wu, Y. Liu, Fixed point theorems for monotone operators and applications to nonlinear elliptic problems, Fixed Point Theory Appl., 134(2013), 14 pages.

Received: November 7, 2015; Accepted: February 26, 2016. 
